\title{
Is extended-field concurrent chemoradiation an option for radiologic negative paraaortic lymph node, locally advanced cervical cancer?
}

This article was published in the following Dove Press journal:

Cancer Management and Research

9 September 2014

Number of times this article has been viewed

\author{
Mushabbab Al Asiri \\ Mutahir A Tunio' \\ Reham Mohamed ${ }^{2}$ \\ Yasser Bayoumi ${ }^{2}$ \\ Abdulrehman Alhadab' \\ Rasha M Saleh ${ }^{3}$ \\ Muhannad Saud AlArifi' \\ Abdelaziz Alobaid ${ }^{4}$ \\ 'Radiation Oncology, Comprehensive \\ Cancer Center, King Fahad Medical \\ City, Riyadh, Saudi Arabia; ${ }^{2}$ Radiation \\ Oncology, National Cancer Institute, \\ Cairo University, Cairo, Egypt; \\ ${ }^{3}$ Medical Oncology, Comprehensive \\ Cancer Center, King Fahad Medical \\ City, Riyadh, Saudi Arabia; ${ }^{4}$ Women's \\ Specialized Hospital, King Fahad \\ Medical City, Riyadh, Saudi Arabia
}

Correspondence: Mutahir Tunio

Radiation Oncology, Comprehensive

Cancer Center, King Fahad Medical City,

Riyadh 59046, Saudi Arabia

Tel +966 | 2889999

Fax +966 | 46| 4006

Emaildrmutahirtonio@hotmail.com
Background: The aim was to evaluate whether extended-field concurrent chemoradiation (EF-CCRT) leads to results better than those obtained by standard whole-pelvis concurrent chemoradiation (WP-CCRT) in locally advanced cervical cancer with radiologic negative paraaortic lymph nodes (PALNs).

Patients and methods: A total of 102 patients with histopathologically proven squamous cell carcinoma, adenocarcinoma, or adenosquamous cell carcinoma, and radiologic negative PALN locally advanced cervical cancer, stage IIB-IVA, were accrued between July 2007 and April 2008 and were randomly assigned to WP-CCRT (50 patients) or EF-CCRT (52 patients), followed by high-dose rate brachytherapy. Data regarding the safety profile, response rates, and occurrence of local, PALN, or distant failure were recorded.

Results: During a median follow-up time of 60 months (18-66), 74/102 patients completed the treatment protocol and were analyzed. Overall PALN, distant-metastasis control, diseasefree survival, and overall survival rates were $97.1 \%, 86.9 \%, 80.3 \%$, and $72.4 \%$ in EF-CCRT respectively in comparison with WP-CCRT $(82.1 \%, 74.7 \%, 69.1 \%$, and $60.4 \%)$, with $P$-values of $0.02,0.03,0.03$ and 0.04 respectively. No difference in acute toxicity profile was seen between the groups, and late toxicities were mild and minimal.

Conclusion: Prophylactic EF-CCRT can be a reasonable option in patients with locally advanced cervical cancer with radiologic positive pelvic lymph nodes and radiologic negative PALN.

Keywords: prophylactic extended field radiation therapy, concurrent chemotherapy

\section{Introduction}

The incidence of invasive cervical cancer is decreasing in United States. ${ }^{1}$ However, cervical cancer continues to be a major women's health issue in many countries because of inadequate cytological screening programs, and many patients present as locally advanced International Federation of Gynecology and Obstetrics (FIGO) stage IIB-IVA, for which surgery is inadequate. The standard treatment for locally advanced cervical cancer is combined chemotherapy and pelvic irradiation, based on results of four randomized trials showing survival benefit of 10\%-15\% and local and distant recurrence reduction rates of $30 \%-40 \%{ }^{2-5}$ However, $15 \%-25 \%$ of patients experience paraaortic lymph nodes (PALN) failures after receiving combined pelvic chemoradiation, in long-term follow up. ${ }^{6}$ Data suggests that patients with locally advanced cervical cancer harbor $17 \%-37 \%$ paraaortic nodal micrometastases, at the time of diagnosis, just outside of standard radiation fields; therefore, the paraaortic nodal area is a common extrapelvic site of failure, which certainly compromises the distant control and survival. ${ }^{7}$ 
Since the last three decades, clinical trials of radiotherapy alone have failed to document the efficacy of prophylactic paraaortic irradiation or extended-field irradiation for biopsy-proven PALNs metastases, PALN failures with 5-year survival rates as low as $29 \%$ to $50 \% .{ }^{8}$ Further observations of chemotherapy incorporated with extended-field irradiation for biopsy-proven PALN metastases showed significant gastrointestinal and hematological toxicities and no survival benefit. ${ }^{9}$

We aimed to evaluate whether prophylactic extended-field irradiation with concurrent chemotherapy leads to results better than those obtained by standard pelvic irradiation with concurrent chemotherapy in patients with locally advanced cervical cancer with radiologic negative PALN status.

\section{Materials and methods Eligibility}

After getting the approval from Institutional (King Fahad Medical City, Riyadh Saudi Arabia) and National ethical review committees (King Abdulaziz City for Science and Technology) and obtaining informed consents from patients for participation in the study in March 2007, consecutive patients with locally advanced cervical cancer, between July 2007 and April 2008, were accrued when they met the following eligibility criteria: 1) histologically proven squamous cell carcinoma, adenocarcinoma or adenosquamous carcinoma of the cervix uteri; 2) clinical and radiologic FIGO stage IIB-IVA, with no other evidence of distant metastasis outside the pelvis; 3) Eastern Cooperative Oncology Group (ECOG) performance status $0-2$; 4) negative PALNs on imaging (computed tomography [CT], magnetic resonance imaging [MRI]), or flourodeoxyglucose positron emission tomography (FDG-PET); and 5) normal hematologic, hepatic, and renal functions tests (white blood cell count $[\mathrm{WBC}] \geq 4,000 / \mathrm{mm}^{3}$, absolute neutrophil count $\left[\right.$ ANC] $\geq 1,500 / \mathrm{mm}^{3}$, platelets $\geq 100,000 / \mathrm{mm}^{3}$, total bilirubin $\leq 1.5 \mathrm{mg} / \mathrm{dL}$, alanine transaminase $\leq 2$ of normal and creatinine $\leq 1.5 \mathrm{mg} / \mathrm{dL}$ ).

Patients with a previous history of hysterectomy, retroperitoneal surgery, abdominal or pelvic radiotherapy, prior chemotherapy, pregnancy, or positive paraaortic nodes on imaging or biopsy proven were excluded.

The pretreatment workup consisted of a complete history and physical examination; bimanual examination; CT and MRI of chest, abdomen, and pelvis; complete hematology, hepatic, renal, and electrolytes studies; bone scan; and cystoscopy and sigmoidoscopy when necessary.

\section{Treatment protocol}

\section{External beam radiation therapy (EBRT)}

All patients were scanned, for simulation, on a CT simulator, from epigastrium to midthighs, and noncontrast and contrast images were obtained. After the data acquisition, all patients were randomized into two groups according to radiation therapy techniques. In the first group, whole-pelvis concurrent chemoradiation (WP-CCRT), the following were delineated: gross tumor volume (GTV); uterus; the presacral, common iliac, internal iliac, and external iliac lymph nodes; initial clinical target volume (CTV-1), covering GTV and the uterus, with $1 \mathrm{~cm}$ margins, and the presacral, common iliac, internal iliac, and external iliac lymph nodes; initial planning target volume (PTV)-1, with 0.5 to $1 \mathrm{~cm}$ margins around CTV-1; boost clinical target volume (CTV-2), covering the positive lymph nodes and parametria; PTV-2 (4 cm midline block and $0.5 \mathrm{~cm}$ margins to CTV2); and organs at risk, including kidneys, small bowel, bladder, rectum, and femoral heads. For the majority of patients; equally spaced, coplanar three-dimensional chemoradiation (3D-CRT) field plans (box-field) were generated; however, intensity-modulated radiation therapy (IMRT) was also generated for some cases, to achieve better dose distribution. Radiation (3D-CRT) was delivered with anteroposterior, posteroanterior, and opposed lateral beams of 18-MV photons. The borders of (anteroposterior/posteroanterior) fields were kept, cranially, at the junction of L3/L4; caudally, $3 \mathrm{~cm}$ below the most inferior vaginal involvement (as marked by golden seeds); and laterally, $2 \mathrm{~cm}$ lateral to the pelvic brim. The borders of lateral fields were kept, anteriorly, $1 \mathrm{~cm}$ anterior to the symphysis pubis, and posteriorly, were extended to the sacrum hollow. The prescribed radiation doses were $45-50.4 \mathrm{~Gy} / 25-28$ fractions to PTV-1, and 54-59.4 Gy/30-33 fractions to PTV-2, 5 days per week, and up to $7 \%$ variation was considered acceptable (Figures 1 and 2A). Radiologic positive lymph nodes were boosted to a dose of 50.4-54 Gy. During planning, the mean dose to the rectum was constrained to $<50 \mathrm{~Gy}$, and the total doses to the small bowel, kidneys, and bladder were constrained to $<45 \mathrm{~Gy},<20 \mathrm{~Gy}$, and $<60$ Gy respectively. In the second group, receiving prophylactic extended-field concurrent chemoradiation (EF-CCRT), the pelvis was treated similarly as for the WP-CCRT group, and additional paraaortic fields were added as a continuous area or with a half-beam block, with a superior field border at the junction of T12/L1, to cover PALNs up to the level of the renal hila. In no cases were anteroposterior or posteroanterior fields allowed, to minimize the toxicity. The prescribed radiation 

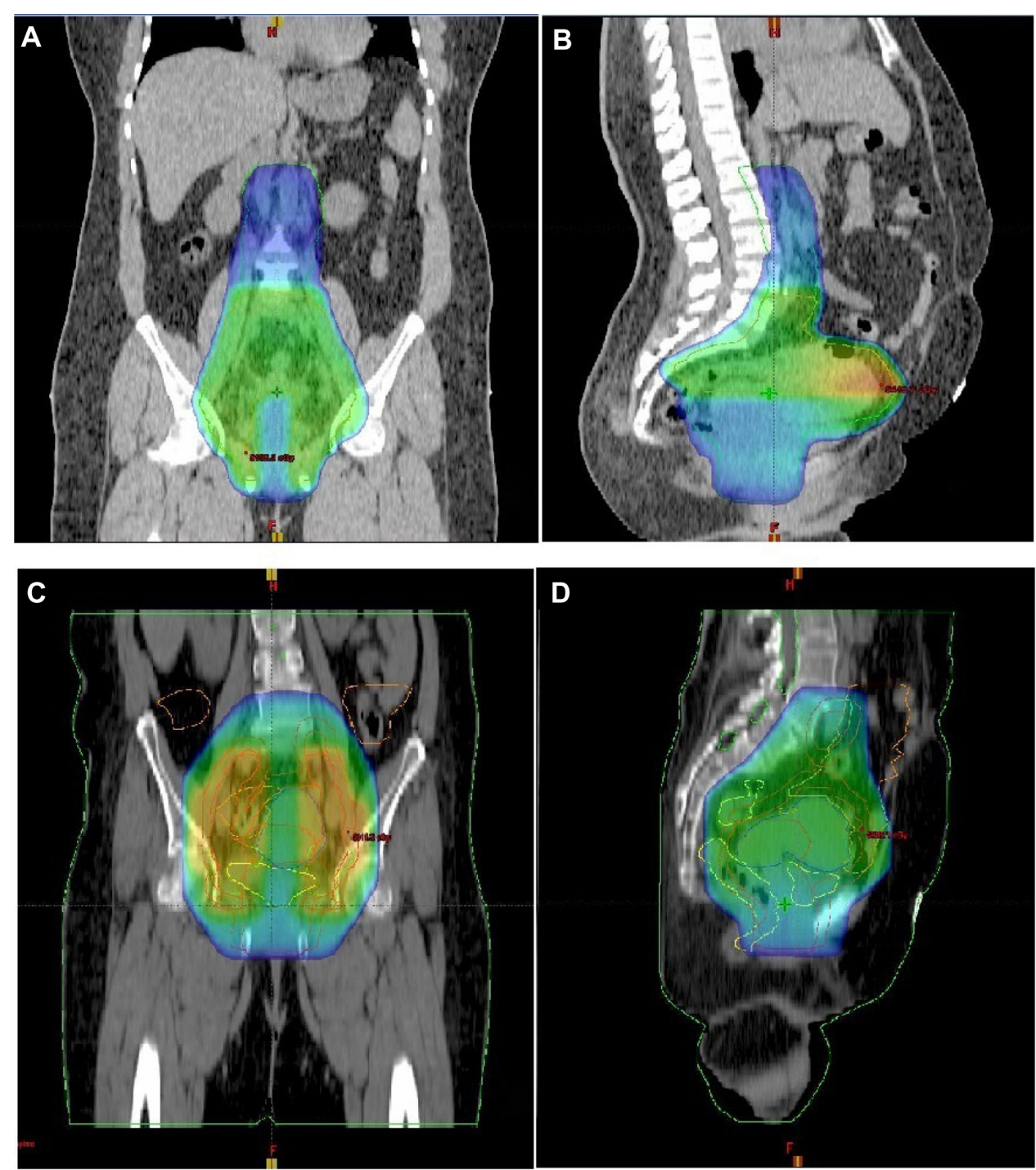

Figure I Initial-phase radiotherapy: (A,B) extended-field and (C,D) three-dimensional conformal radiation therapy (3D-CRT) plans showing 45 Gy to planning target volume (PTV)-I, in coronal $(\mathbf{A}, \mathbf{C})$ and sagittal views (B,D).

dose to PALNs was 45 Gy (up to 7\% variation was considered acceptable). All patients in the EF-CCRT arm were given routine antiemetics before planned radiotherapy sessions.

\section{Concurrent chemotherapy}

Both groups of patients received weekly cisplatin $40 \mathrm{mg} / \mathrm{m}^{2}$ before the administration of radiotherapy for six doses. During CCRT, the doses were modified on a weekly basis. If the WBC count was below $2,000 / \mathrm{mm}^{3}$ or the platelet count was below $50,000 / \mathrm{mm}^{3}$, all chemotherapy for that week was omitted. If the WBC count was below $1,000 / \mathrm{mm}^{3}$ or the platelet count was below $25,000 / \mathrm{mm}^{3}$, CCRT was withheld until the WBC count and the platelet count recovered to $1,000 / \mathrm{mm}^{3}$ or greater and $25,000 / \mathrm{mm}^{3}$ or greater, respectively. If the serum creatinine level was above $1.8 \mathrm{mg} / \mathrm{dL}$, the cisplatin was withheld. Patients were deemed unsuitable for further chemotherapy if the delay to resume treatment was longer than 2 weeks.

\section{High-dose rate (HDR) brachytherapy}

Fletcher-Suit tandem and ovoids afterloading applicators were used for HDR-brachytherapy with iridium-192 sources, once a week under conscious sedation, after 45 Gy of EBRT. A dose of 7 Gy per fraction, using three insertions to point A with a total dose of $21 \mathrm{~Gy}$, was delivered, based on the dose limit derived from the treatment plan for the rectum and bladder. The dose constraints were $75 \mathrm{~Gy}$ and $80 \mathrm{~Gy}$ for the rectum and bladder, respectively (Figure 2B). 


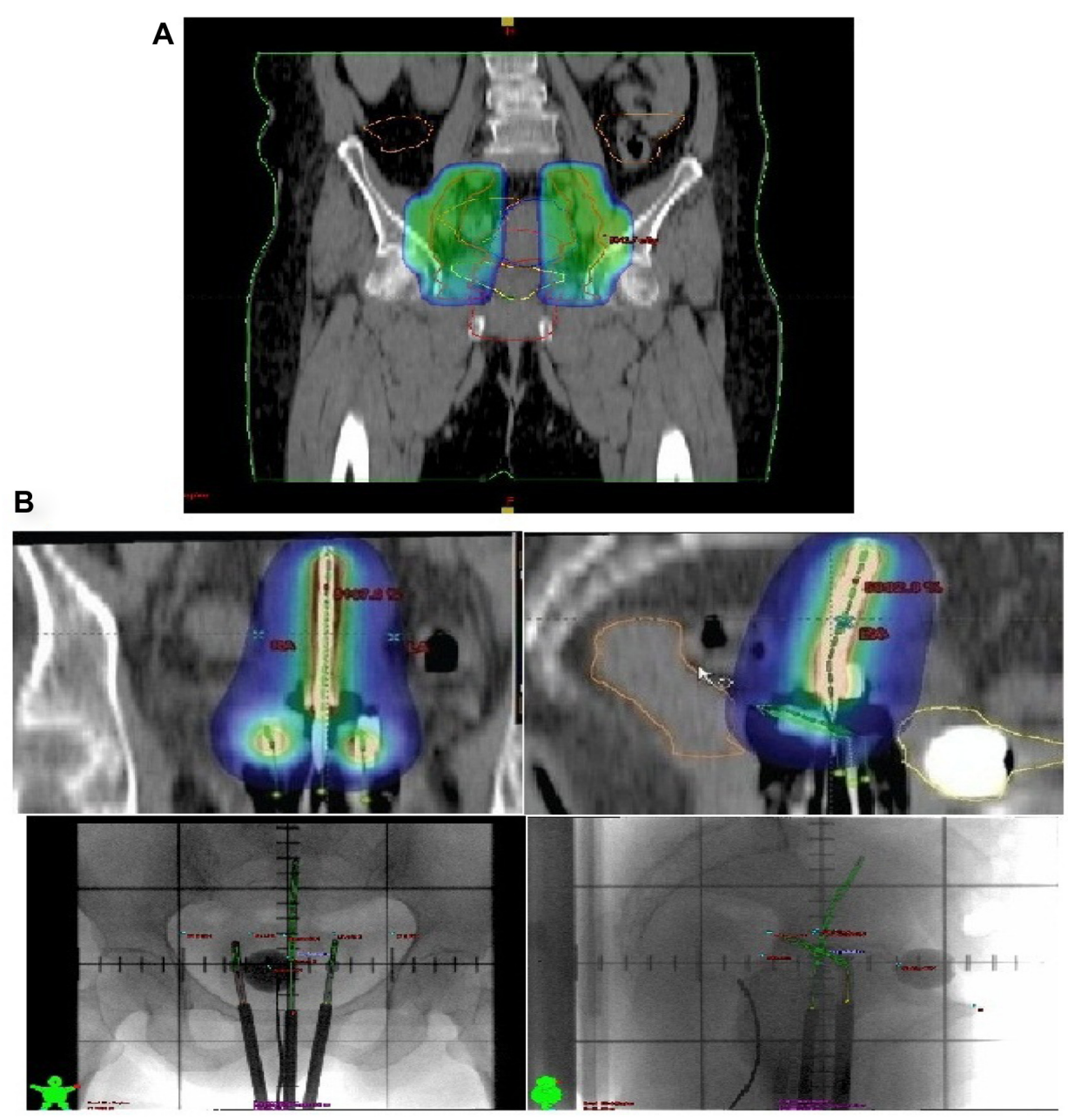

Figure 2 (A) Parametrial boost (planning target volume [PTV]-2) of 5.4-9 Gy with midline shielding, followed by (B) high-dose rate brachytherapy of 2 I Gy in three sessions, in the two treatment groups (rectum on the left and bladder on the right).

\section{Toxicity scoring}

During CCRT, patients were evaluated every week for weight, performance status, pelvic examination, hematologic and blood chemistry determinations, and other related characteristics. The National Cancer Institute Common Toxicity Criteria (NCI-CTC) version 2.0 were used to score acute radiation and chemotherapy toxicity $(<90$ days from the start of radiation therapy).

After completion of the CCRT, patients were seen every 3 months for the first 2 years, and every 6 months thereafter, at radiation oncology and gynecology oncology clinics. Response evaluation consisted of physical and pelvic examination; Pap smear; hematology, hepatic, and renal function tests; and CT chest, and abdomen and pelvic MRI every 6 months for the first 2 years. The Radiation Therapy Oncology Group (RTOG) Late Radiation Morbidity Scoring Criteria were used to score radiation toxicity persisting beyond 90 days from the completion of radiotherapy.

\section{Statistical analysis}

The study was initially designed to be able to detect an improvement of approximately $10 \%$ in the 5 -year locoregional control (LRC), with a statistical power of $80 \%$ and a two-sided significance level of 0.05 . To detect such a difference, we predicted that we would need to enroll 163 women in the study over a 2-year period and then follow them for an additional 4 years. We estimated that $50 \%$ (86) women would have died by the time of the analysis. The primary end point was the LRC (pelvic and paraaortic control). Secondary end points were distant metastatic control, disease-free survival (DFS), and the overall survival (OS) for each group. DFS was defined as the duration between the completion of CCRT and the date of documented disease recurrence, death resulting from the cancer, and/or last follow-up visit (censored). OS was defined as the duration between the completion of CCRT and the date of patient death or last follow-up visit (censored). The probabilities of LRC, distant metastatic control, DFS, 
and OS were determined with the Kaplan-Meier method. The comparisons for various end points were performed using the log-rank test. The Student's unpaired $t$ test was used to determine the significance of the difference between two groups. A $P$-value of 0.05 was considered statistically significant. Statistical analyses were performed using the computer program SPSS (Statistical Package for the Social Sciences, Version 17.0; IBM Corp, Chicago, IL, USA).

\section{Results}

\section{Characteristics of the patients}

After accrual, 83 patients with locally advanced cervical cancer were randomly assigned to WP-CCRT (50 patients) and EF-CCRT (52 patients), as shown in Figure 3. The characteristics of remaining 74 patients are given in Table 1 . The majority of the cohort was presented with FIGO stage IIB (66.2\%) and with radiologic positive pelvic lymph nodes (51.4\%). FIGO stage IIIB with hydronephrosis/ nonfunctioning kidneys was seen in three cases $(4.05 \%)$, and for these patients, percutaneous nephrostomy or JJ stenting was performed prior to starting CCRT. The median follow-up time was 60 months (range, 18-66). The histopathologic characteristics of patients with positive pelvic lymph nodes are shown in Table 2.

Among all 74 patients who received CCRT, the treatment protocol completion rate was 90\% (95\% confidence interval [CI]: $85-100)$ in the WP-CCRT group and $88.4 \%$ (95\% CI: 90-100) in the EF-CCRT group $(P=0.8)$. Weekly concurrent cycles of cisplatin, in both treatment arms, were completed in all $74(100 \%)$ patients, with no interruption. The median duration of radiation therapy (including EBRT and

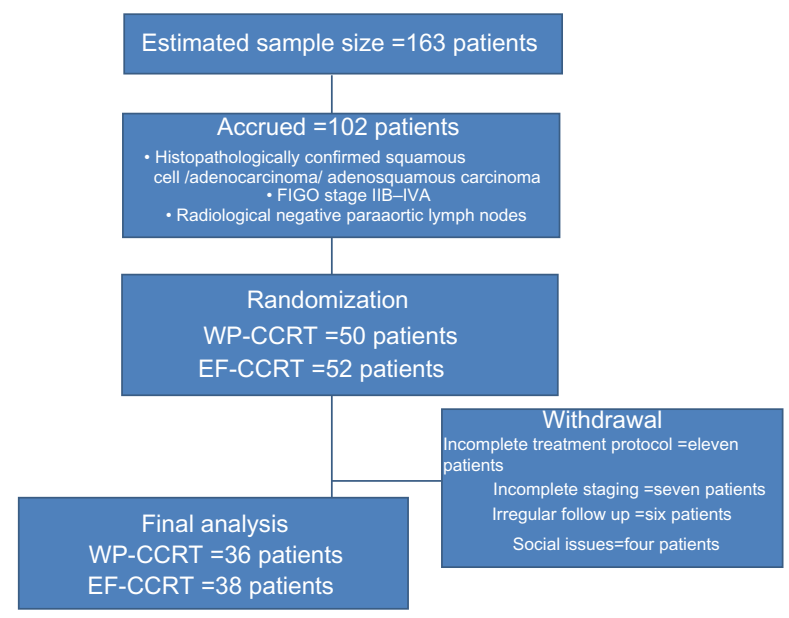

Figure 3 Treatment algorithm.

Abbreviations: EF-CCRT, extended-field concurrent chemoradiation; FIGO, International Federation of Gynecology and Obstetrics; WP-CCRT, whole-pelvis concurrent chemoradiation.
HDR-brachytherapy) in both arms was 55.5 days ( 7.8 weeks) with 95\% CI: 48-58.

\section{Toxicity profile}

The overall incidence of the grade 3 or 4 acute hematological and nonhematological toxicities was 5.2\% and 2.6\%, respectively, in the EF-CCRT arm and $5.4 \%$ and $2.7 \%$, respectively, in the WP-CCRT $\operatorname{arm}(P=0.7)$. Both treatment arms had similar grade 3 or 4 acute gastrointestinal toxicity (Table 3 ).

During the follow-up time of 60 months, in the EF-CCRT group, one patient $(2.6 \%)$ experienced subacute intestinal obstruction. No patient in either arm underwent surgery for radiation-induced damage or died because of treatmentrelated side effects.

\section{Pelvic, paraaortic, distant control, and survival rates}

At the time of the last follow-up visit, three (7.9\%) patients had pelvic recurrences (one vaginal and two pelvic nodal) in the EF-CCRT arm, and three (8.3\%) patients had local recurrences (pelvic nodal) in the WP-CCRT arm $(P=0.8)$. Isolated vaginal recurrence in the EF-CCRT group was successfully salvaged with surgery, and pelvic nodal recurrences were treated with salvage chemotherapy. First pelvic recurrence was manifested within 24 months of completion of CCRT in both arms.

Paraaortic nodal failures were seen in five (13.9\%) patients in the WP-CCRT arm and one (2.6\%) patient in EFCCRT $\operatorname{arm}(P=0.02)$ (Figure 3A). In the WP-CCRT arm, three $(60 \%)$ of five paraaortic nodal failures were seen at the level of junction L2 and L3 vertebrae and two (40\%) at the level of L1 vertebrae; and in the EF-CCRT arm, one paraaortic nodal failure was seen at the junction of T12 and L1 vertebrae. In both arms, paraaortic nodal failures were seen within 20 months of completion of CCRT. Distant failures were seen in seven $(19.4 \%)$ patients in the WP-CCRT and in four $(10.5 \%)$ patients from the EF-CCRT arm $(P=0.02)$ (Figure 4B). The common sites of distant failure were: lungs (four patients), mediastinal (three patients), and supraclavicular nodes (one patient), and thoracic spine (one patient). Combined distant and paraaortic nodal failures were observed in four $(11.4 \%)$ in the WP-CCRT and one (2.6\%) in the EF-CCRT group. All distant and PALN were treated with salvage chemotherapy \pm radiotherapy for bony lesions. Important prognostic factors for PALN were positive pelvic nodes on imaging (CT/ MRI), the FIGO stage $>$ IIB, and prolonged treatment time $>55$ days ( $P=0.001,0.001$ and 0.03 , respectively) (Table 4$)$. At the time of analysis, 32 patients in the EF-CCRT and 27 
Table I Patients characteristics

\begin{tabular}{|c|c|c|c|}
\hline Variables & $\begin{array}{l}\text { Arm A } \\
\text { Extended-field CCRT } \\
(n=38)\end{array}$ & $\begin{array}{l}\text { Arm B } \\
\text { Pelvic-field CCRT } \\
(n=36)\end{array}$ & $P$-value \\
\hline Mean age & 52.3 years $(32-78)$ & 51.6 years $(34-76)$ & 0.9 \\
\hline ECOG performance scale & $0-2$ & $0-2$ & 1.0 \\
\hline \multicolumn{4}{|l|}{ Histopathology } \\
\hline Squamous cell carcinoma & $34(89.5 \%)$ & 33 (91.7\%) & 0.7 \\
\hline Adenocarcinoma & $3(7.9 \%)$ & $2(5.6 \%)$ & \\
\hline Adenosquamous cell carcinoma & I (2.6\%) & I (2.8\%) & \\
\hline \multicolumn{4}{|l|}{ FIGO staging } \\
\hline IIB & $24(63.1 \%)$ & 25 (69.4\%) & 0.8 \\
\hline IIIA & $6(15.8 \%)$ & $6(16.6 \%)$ & \\
\hline IIIB & $4(10.5 \%)$ & $3(8.3 \%)$ & \\
\hline IVA & $4(10.5 \%)$ & $2(5.6 \%)$ & \\
\hline \multicolumn{4}{|l|}{ Radiological primary tumor size } \\
\hline$<5 \mathrm{~cm}$ & $13(34.2 \%)$ & 14 (38.9\%) & 0.6 \\
\hline$>5 \mathrm{~cm}$ & $25(65.8 \%)$ & $22(61.1 \%)$ & \\
\hline \multicolumn{4}{|l|}{ MRI-based nodal involvement } \\
\hline Negative & $15(39.5 \%)$ & $21(58.4 \%)$ & 0.05 \\
\hline Iliac & $9(23.7 \%)$ & $13(36.1 \%)$ & \\
\hline Common Iliac & $14(36.8 \%)$ & $2(5.6 \%)$ & \\
\hline Paraaortic & - & - & \\
\hline \multicolumn{4}{|l|}{ Pretreatment hemoglobin } \\
\hline$>10 \mathrm{gm} / \mathrm{dL}$ & $35(92.1 \%)$ & 34 (94.4\%) & 0.9 \\
\hline$<10 \mathrm{gm} / \mathrm{dL}$ & $3(7.9 \%)$ & $2(5.6 \%)$ & \\
\hline \multicolumn{4}{|l|}{ Treatment } \\
\hline 3D-CRT & 30 (78.9\%) & $36(100.0 \%)$ & 0.9 \\
\hline IMRT & $8(21.1 \%)$ & - & \\
\hline \multicolumn{4}{|l|}{ EBRT } \\
\hline Whole pelvis & 45 Gy (42-50.4) & 45 Gy (42-50.4) & \\
\hline Paraaortic & 45 Gy (45-50.4) & - & \\
\hline Parametrial/positive $L N$ boost & 9 Gy (5-9) & 9 Gy (5-9) & \\
\hline \multicolumn{4}{|l|}{ HDR-BT } \\
\hline Dose/fraction & 7 Gy/fraction & 7 Gy/fraction & \\
\hline Total dose/fraction & $21 \mathrm{~Gy} / 3$ & $21 \mathrm{~Gy} / 3$ & \\
\hline Point A BED & 86.4 Gy (80.5-102.7) & 86.4 Gy $(80.5-102.7)$ & \\
\hline ICRU 38 rectal point BED & 85 Gy $(80.5-100)$ & 85 Gy $(80.5-100)$ & \\
\hline ICRU 38 bladder point BED & 86 Gy (80.5-102) & 86 Gy $(80.5-102)$ & \\
\hline \multicolumn{4}{|l|}{ Concurrent weekly cisplatin cycle } \\
\hline Dose/week & $30 \mathrm{mg} / \mathrm{m}^{2}$ & $30 \mathrm{mg} / \mathrm{m}^{2}$ & \\
\hline Mean cycles & $5(4-7)$ & $5(4-7)$ & \\
\hline
\end{tabular}

Abbreviations: 3D-CRT, three-dimensional conformal radiation therapy; BED, biologic effective dose; CCRT, concurrent chemoradiation; EBRT, external beam radiation therapy; ECOG, European Cooperative Oncology Group; FIGO, International Federation of Gynecologists and Obstetricians; HDR-BT, high-dose rate brachytherapy; ICRU, international commission of radiation units; IMRT, intensity modulated radiation therapy; LN, lymph nodes; MRI, magnetic resonance imaging.

patients in the WP-CCRT group were found without evidence of disease (Figure 4C and D).

\section{Discussion}

WP-CCRT is deemed the standard treatment, by many North American and European teams, for treatment of locally advanced cervical cancer, which has resulted in survival gain but also, 10\%-25\% paraaortic nodal failures. ${ }^{10}$ The 10 -year results of a RTOG (RTOG-97-20) trial, which compared pelvic irradiation with paraaortic plus pelvic radiotherapy alone, have shown an improvement in survival gain of $11 \%$ in the prophylactic paraaortic plus pelvic irradiation group but no difference in LRC. ${ }^{11,12}$ A subsequent other RTOG trial (RTOG 90-01) compared prophylactic extended-field radiotherapy versus pelvic irradiation with chemotherapy in locally advanced cervical cancer patients; the study showed that 5-year OS rates for patients treated with pelvic irradiation and concurrent chemotherapy was significantly greater than for patients treated with extended-field radiation alone ( $67 \%$ vs $41 \%$ at 8 years), and pelvic irradiation with concurrent chemotherapy resulted in a $51 \%$ reduction in the risk of recurrence and a $52 \%$ reduction in the risk of death but 
Table 2 Clinicopathological findings variation in cervical patients with positive lymph nodes

\begin{tabular}{|c|c|c|c|}
\hline Variables & $\begin{array}{l}\text { Negative } \\
\text { lymph nodes } \\
(n=36)\end{array}$ & $\begin{array}{l}\text { Metastatic } \\
\text { lymph nodes } \\
(n=38)\end{array}$ & $P$-value \\
\hline Age (range) & $\begin{array}{l}51.6 \text { years } \\
(44-76)\end{array}$ & $\begin{array}{l}47.3 \text { years } \\
(32-57)\end{array}$ & 0.04 \\
\hline ECOG performance scale & $0-2$ & $0-2$ & 1.0 \\
\hline \multicolumn{4}{|l|}{ Histopathology } \\
\hline Squamous cell carcinoma & 33 (9l.7\%) & $34(89.5 \%)$ & 0.6 \\
\hline Keratinizing & $8(24.3 \%)$ & $10(29.4 \%)$ & \\
\hline Nonkeratinizing & $25(75.7 \%)$ & $24(70.6 \%)$ & \\
\hline Adenocarcinoma & $4(11.1 \%)$ & $\mathrm{I}(2.6 \%)$ & 0.003 \\
\hline $\begin{array}{l}\text { Adenosquamous } \\
\text { carcinoma }\end{array}$ & 0 & $2(5.3 \%)$ & 0.04 \\
\hline \multicolumn{4}{|l|}{ FIGO staging } \\
\hline IIB & 34 (94.4\%) & $15(39.4 \%)$ & 0.003 \\
\hline IIIA & $\mathrm{I}(2.8 \%)$ & II (28.9\%) & \\
\hline IIIB & I (2.8\%) & $6(15.8 \%)$ & \\
\hline IVA & $0(0 \%)$ & $6(15.8 \%)$ & \\
\hline \multicolumn{4}{|c|}{ Radiological primary tumor size } \\
\hline$<5 \mathrm{~cm}$ & $17(47.2 \%)$ & $10(26.3 \%)$ & 0.04 \\
\hline$>5 \mathrm{~cm}$ & $19(52.8 \%)$ & $28(73.7 \%)$ & \\
\hline \multicolumn{4}{|l|}{ Uterine corpus invasion } \\
\hline No & $29(80.6 \%)$ & $13(34.2 \%)$ & 0.003 \\
\hline Yes & $7(19.4 \%)$ & 25 (65.8\%) & \\
\hline \multicolumn{4}{|l|}{ Vaginal invasion } \\
\hline No & 27 (75.0\%) & $12(31.6 \%)$ & 0.004 \\
\hline Yes & $9(25.0 \%)$ & $26(68.4 \%)$ & \\
\hline
\end{tabular}

Abbreviations: ECOG, European Cooperative Oncology Group; FIGO, International Federation of Gynecologists and Obstetricians.

no difference in the incidence of paraaortic failures $(7 \%$ in pelvic irradiation with concurrent chemotherapy vs $4 \%$ in extended-field irradiation $[P$-value 0.15$]){ }^{6}$

The role of incorporating concurrent chemotherapy with prophylactic extended-field irradiation to reduce the paraaortic nodal failures and promote a survival gain is not well known; due to lack of Phase III trials to pursue this question, Malfetano et al used weekly cisplatin concomitant with prophylactic extended-field radiation, followed by low-dose rate brachytherapy, in a Phase II study in which 67 women were enrolled, of whom 54 had radiologic negative paraaortic nodes. Treatment was well tolerated, with high efficacy, and at a median follow up of 47.5 months, $75 \%$ of patients were alive without evidence of disease and with no paraaortic failures. ${ }^{13}$ However, this trial was criticized for nonrandomization, selection bias, and use of older conventional external beam and low-dose rate radiation techniques. Another Phase II study by Chung et al treated 63 locally advanced cervical cancers with concurrent cisplatin during the first and fifth weeks of extended-field irradiation, followed by two adjuvant cycles of cisplatin- and 5-flouro-uracil-based chemotherapy; results showed that toxicity was minimal, with paraaortic nodal failures in only $4.5 \%$ and an OS rate of $81 \%$ at 3 years. ${ }^{14}$ Advanced FIGO stage, parametrial invasion, elevated CEA, and SCC-Ag are all associated with increased risk of PALN failure.

To the best of our knowledge, our single institutional study is the first randomized, prospective study to compare prophylactic EF-CCRT plus HDR brachytherapy with standard WP-CCRT plus HDR brachytherapy, in patients with locally advanced cervical cancer with radiologic negative PALNs. We used radiologic (CT/MRI) staging along with clinical FIGO staging, as tumor size and lymph node involvement are also important prognostic factors that influence the LRC and survival. ${ }^{17,18}$ For such patients, CT/PET imaging would be superior to other imaging modalities to assess the lymph node status and distant metastasis; ${ }^{19}$ however, our study can be criticized for not performing baseline CT/PET in all patients. Possible explanation was the nonavailability of PET imaging in our institute at the time of accrual of patients (and later on, only $16 \%$ of patients had routine follow-up CT/PET imaging). The course of treatment was well tolerated in our patients, and hematological and nonhematological toxicities were minimal or even better than those reported in other trials of EF-CCRT., ${ }^{4,5}$ Further, EF-CCRT resulted in excellent 5-year paraaortic distant control rates and better DFS and OS rates, in comparison with WP-CCRT, in the present study, especially in patients with radiologic gross pelvic lymphadenopathy; however, we did not see any difference in pelvic control between the two groups.

Possible explanation for the lower toxicity profile and better outcomes (LRC and distant control) in the EF-CCRT group may be that: a) the majority of our patients (90\%) had gross pelvic nodes $>1 \mathrm{~cm}$ on imaging, which enhances the risk of paraaortic nodal micrometastasis, and these were eradicated by prophylactic EF-CCRT; b) surgical staging causes more intestinal fibrosis which results in more gastrointestinal toxicity; c) moderate doses of weekly cisplatin were used than elsewhere; d) use of better radiation techniques in our study (CT simulation, 3D-CRT/IMRT and HDR brachytherapy); and e) treatment duration was 55 days for the majority of patients.

Although our study showed encouraging treatment outcomes and low morbidity, limitations of the present study were: a) the low sample size; b) poor randomization to study treatment and selection bias as many patients with common iliac lymphadenopathy were included in the EFCCRT arm; c) lack of baseline FDG-PET-based staging; and e) the lack of baseline carcinoembryonic antigen and SCC-Ag levels. 
Table 3 Acute and late treatment related toxicity profile

\begin{tabular}{|c|c|c|c|c|c|}
\hline \multirow[t]{2}{*}{ Toxicity } & \multicolumn{2}{|c|}{$\begin{array}{l}\text { Arm A } \\
\text { Extended-field CCRT } \\
(n=38)\end{array}$} & \multicolumn{2}{|c|}{$\begin{array}{l}\text { Arm B } \\
\text { WP CCRT } \\
(n=36)\end{array}$} & \multirow[t]{2}{*}{$P$-value } \\
\hline & G3 & G4 & G3 & G4 & \\
\hline \multicolumn{6}{|l|}{ Acute } \\
\hline \multicolumn{6}{|l|}{ Hematologic } \\
\hline Neutropenia & I (2.6\%) & I (2.6\%) & I (2.7\%) & I (2.7\%) & \\
\hline Thrombocytopenia & 0 & 0 & 0 & 0 & \\
\hline Anemia & 0 & 0 & 0 & 0 & \\
\hline \multicolumn{6}{|l|}{ Nonhematologic } \\
\hline Nausea/vomiting & 0 & 0 & 0 & 0 & 0.7 \\
\hline Diarrhea & I (2.6\%) & 0 & 0 & 0 & \\
\hline Cystitis & 0 & 0 & I (2.7\%) & 0 & \\
\hline Deranged renal functions & 0 & 0 & 0 & 0 & \\
\hline Deranged liver functions & 0 & 0 & 0 & 0 & \\
\hline \multicolumn{6}{|l|}{ Late } \\
\hline Chronic cystitis & 0 & & & & 0.8 \\
\hline Intestinal obstruction & I (2.6\%) & & & & \\
\hline Proctitis & 0 & & & & \\
\hline Neuropathy/plexopathy & 0 & & & & \\
\hline Hearing loss & 0 & & I (2.8\%) & & \\
\hline Renal & 0 & & 0 & & \\
\hline
\end{tabular}

Abbreviations: CCRT, concurrent chemoradiation; WP-CCRT, whole-pelvis concurrent chemoradiation; G, grade.

A

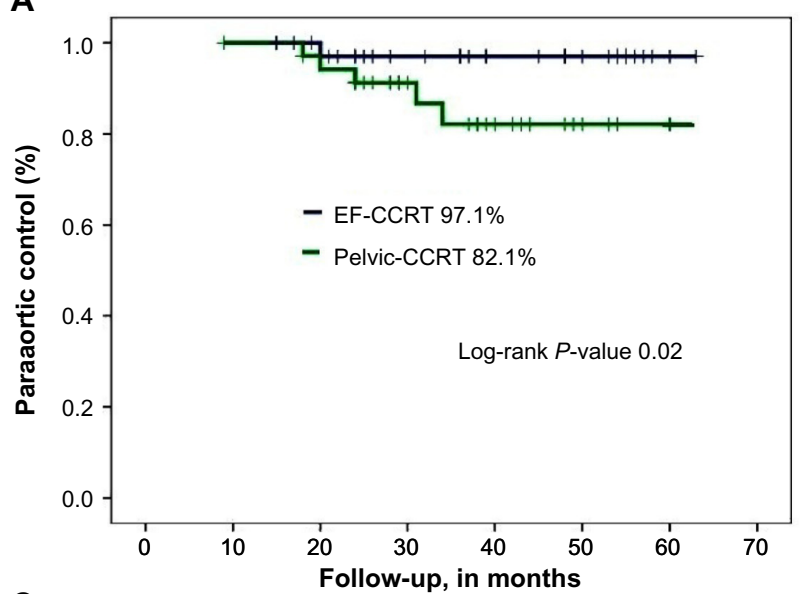

C

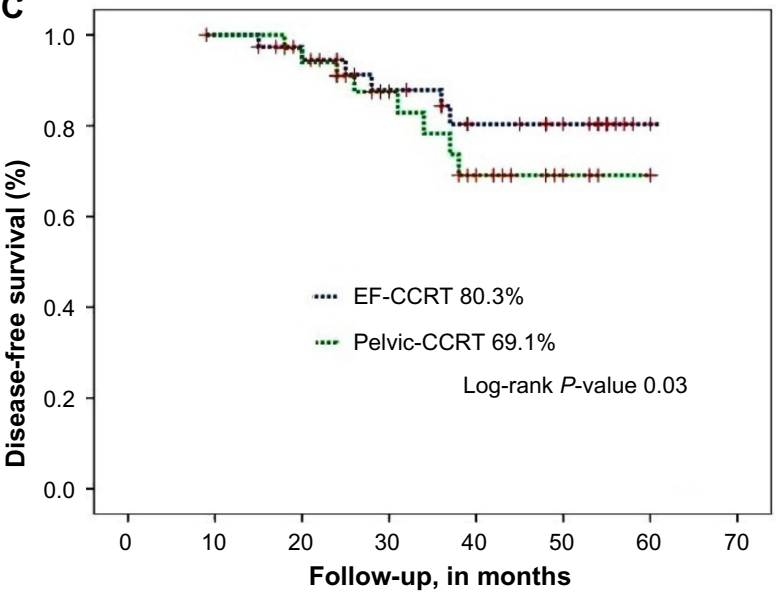

B
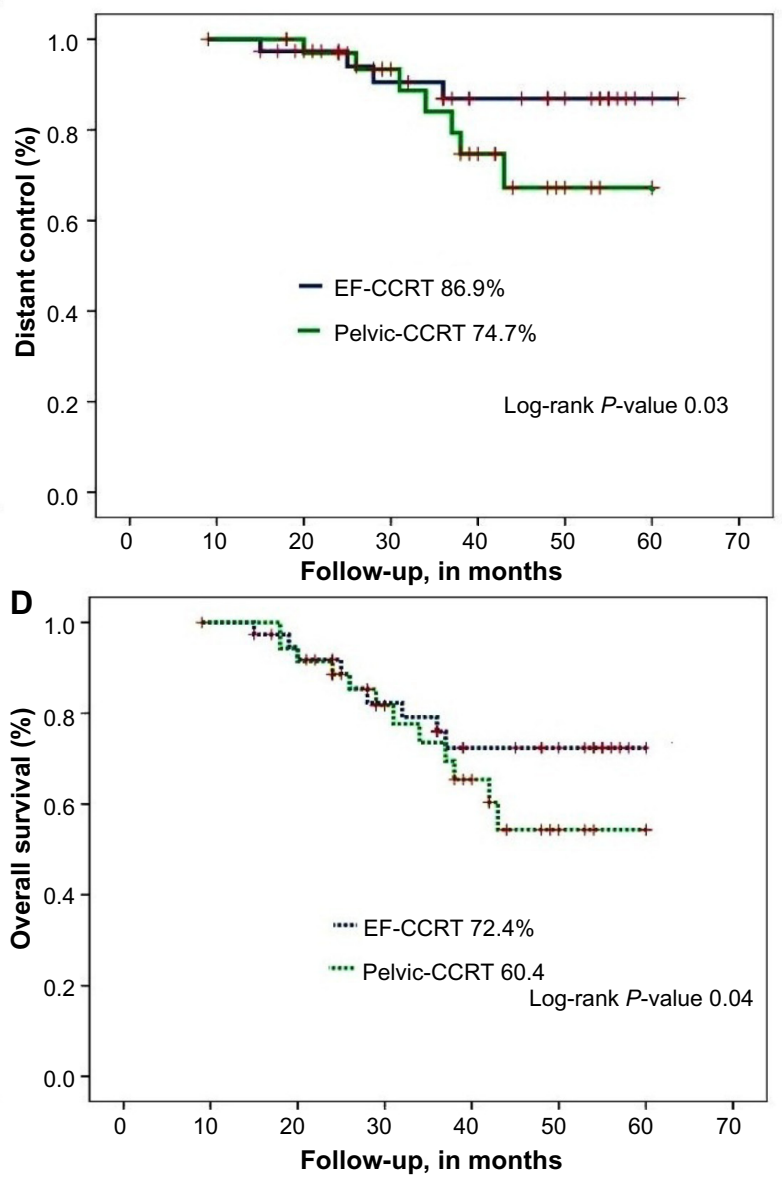

Figure 4 Five year (A) paraaortic control, (B) distant control, (C) disease-free survival, and (D) overall survival, after extended-field chemoradiation and WP-CCRT. Abbreviations: CCRT, concurrent chemoradiation; EF-CCRT, extended-field concurrent chemoradiation; WP-CCRT, whole-pelvis concurrent chemoradiation. 
Table 4 Multivariate analysis of variables on paraaortic, distant control, and disease-free survival rates

\begin{tabular}{|c|c|c|c|}
\hline Variable & $\begin{array}{l}\text { Paraaortic control } \\
\text { OR }(95 \% \mathrm{Cl}) \text { P-value }\end{array}$ & $\begin{array}{l}\text { Distant metastasis control } \\
\text { OR }(95 \% \mathrm{Cl}) \mathrm{P} \text {-value }\end{array}$ & $\begin{array}{l}\text { Disease-free survival } \\
\text { OR }(95 \% \mathrm{CI}) P \text {-value }\end{array}$ \\
\hline Age ( $<50$ vs $>50$ years $)$ & $1.80(0.79-2.10) 0.8$ & $1.10(0.89-2.00) 0.6$ & $0.7 I(0.10-2.4 I) 0.5$ \\
\hline Comorbidities (yes vs no) & $0.88(0.67-0.97) 0.9$ & $1.80(0.79-2.10) 0.7$ & $\mathrm{I} .80(0.79-2.10) 0.7$ \\
\hline FIGO stage $(<$ IIB vs $>$ IIB $)$ & $6.11(2.22-16.30) 0.001$ & $3.65(I .8 I-9.65) 0.02$ & $3.85(1.91-10.35) 0.01$ \\
\hline $\mathrm{N}$ stage (N0 vs $\mathrm{NI})$ & $4.34(3.52-1 \mathrm{I} .34) 0.00 \mathrm{I}$ & $4.01(2.21-11.59) 0.01$ & $2.76(1.75-9.36) 0.03$ \\
\hline Hemoglobin level $(<10$ vs $>10)$ & $2.24(1.65-4.40) 0.03$ & I.I0 (0.89-2.00) 0.5 & $0.78(0.23-2.38) 0.4$ \\
\hline Cell type ( SCC vs non-SCC) & $1.21(1.10-2.10) 0.4$ & $1.10(0.89-2.00) 0.6$ & $1.21(1.10-2.10) 0.7$ \\
\hline EF-CCRT vs WP-CCRT & $3.45(1.61-9.45) 0.02$ & $2.97(1.95-10.5) 0.04$ & $3.65(I .8 I-9.65) 0.02$ \\
\hline Treatment duration ( $<55$ vs $>55$ days $)$ & $2.21(1.45-7.85) 0.03$ & I.I (0.67-I.97) 0.9 & $1.10(0.89-2.00) 0.6$ \\
\hline
\end{tabular}

Abbreviations: $\mathrm{Cl}$, confidence interval; EF-CCRT, extended-field concurrent chemoradiation; FIGO, International Federation of Gynecologists and Obstetricians; OR, odds ratio; SCC, squamous cell carcinoma; WP-CCRT, whole-pelvis concurrent chemoradiation.

However, the results of our study recommend use prophylactic EF-CCRT in patients with locally advanced cervical cancer with radiologic positive pelvic lymph nodes. Further, our results warrant a multicenter, Phase III trial using IMRT with prophylactic EF-CCRT in FDG-PET negative PALN locally advanced cervical patients, to evaluate whether the outcomes of EF-CCRT are truly better than those of WP-CCRT.

\section{Acknowledgment}

We thank Laura Stanciu Gabriella, medical physicist, who did manuscript language editing on behalf of the authors.

\section{Disclosure}

The authors report no conflicts of interest in this work.

\section{References}

1. Siegel R, Naishadham D, Jemal A. Cancer statistics, 2012. CA Cancer $J$ Clin. 2012;62(1):10-29.

2. Keys HM, Bundy BN, Stehman FB, et al. Cisplatin, radiation, and adjuvant hysterectomy compared with radiation and adjuvant hysterectomy for bulky stage IB cervical carcinoma. $N$ Engl $J$ Med. 1999;340(15):1154-1161.

3. Rose PG, Bundy BN, Watkins EB, et al. Concurrent cisplatin based radiotherapy and chemotherapy for locally advanced cervical cancer. N Eng J Med. 1999;340(15):1144-1153.

4. Whitney CW, Sause W, Bundy BN, et al. Randomized comparison of fluorouracil plus cisplatin versus hydroxyurea as an adjunct to radiation therapy in stage IIB-IVA carcinoma of the cervix with negative para-aortic lymph nodes: a Gynecologic Oncology Group and Southwest Oncology Group study. J Clin Oncol. 1999;17(5):1339-1348.

5. Peters WA III, Liu PY, Barrett RJ, et al. Cisplatin and 5-Flourouracil plus radiation therapy are superior to radiation therapy as adjunctive in high risk early stage carcinoma of the cervix after radical hysterectomy and pelvic lymphadenectomy: report of a phase III intergroup study. J Clin Oncol. 2000;18:1606-1613.

6. Eifel PJ, Winter K, Morris M, et al. Pelvic irradiation with concurrent chemotherapy versus pelvic and para-aortic irradiation for high-risk cervical cancer: an update of radiation therapy oncology group trial (RTOG) 90-01. J Clin Oncol. 2004;22(5):872-880.

7. Berman ML, Keys H, Creasman W, DiSaia P, Bundy B, Blessing J. Survival and patterns of recurrence in cervical cancer metastatic to periaortic lymph nodes (a Gynecologic Oncology Group study). Gynecol Oncol. 1984;19(1):8-16.
8. Morris M, Eifel PJ, Lu J, et al. Pelvic radiation with concurrent chemotherapy compared with pelvic and para-aortic radiation for high-risk cervical cancer. N Engl J Med. 1999;340(15):1137-1143.

9. Small W, Winter K, Levenback C, et al. Extended-field irradiation and intracavitary brachytherapy combined with cisplatin chemotherapy for cervical cancer with positive para-aortic or high common iliac lymph nodes: results of ARM 1 of RTOG 0116. Int J Radiat Oncol Biol Phys. 2007;68(4):1081-1087.

10. Gouy S, Morice P, Narducci F, et al. Nodal-staging surgery for locally advanced cervical cancer in the era of PET. Lancet Oncol. 2012;13(5):e212-e220.

11. Rotman M, Choi K, Guse C, et al. Prophylactic irradiation of the paraaortic lymph node chain in stage IIB and bulky stage IB carcinoma of the cervix, initial treatment results of RTOG 7920. Int J Radiat Oncol Biol Phys. 1990;19(3):513-521.

12. Rotman M, Pajak TF, Choi K, et al. Prophylactic extended-field irradiation of para-aortic lymph nodes in stages IIB and bulky IB and IIA cervical carcinomas. Ten-year treatment results of RTOG 79-20. JAMA. 1995;274(5):387-393.

13. Malfetano JH, Keys H, Cunningham MJ, Gibbons S, Ambros R. Extended field radiation and cisplatin for stage IIB and IIIB cervical carcinoma. Gynecol Oncol. 1997;67(2):203-207.

14. Chung YL, Jian JJ, Cheng SH, et al. Extended-field radiotherapy and high-dose-rate brachytherapy with concurrent and adjuvant cisplatinbased chemotherapy for locally advanced cervical cancer: a phase I/II study. Gynecol Oncol. 2005;97(1):126-135.

15. Huang EY, Huang YJ, Chanchien CC, et al. Pretreatment carcinoembryonic antigen level is a risk factor for para-aortic lymph node recurrence in addition to squamous cell carcinoma antigen following definitive concurrent chemoradiotherapy for squamous cell carcinoma of the uterine cervix. Radiat Oncol. 2012;7:13.

16. Jeong BK, Choi DH, Huh SJ, Park W, Bae DS, Kim BG. The role of squamous cell carcinoma antigen as a prognostic and predictive factor in carcinoma of uterine cervix. Radiat Oncol J. 2011;29(3):191-198.

17. Kodaira T, Fuwa N, Toita T, et al. Comparison of prognostic value of MRI and FIGO stage among patients with cervical carcinoma treated with radiotherapy. Int J Radiat Oncol Biol Phys. 2003;56(3):769-777.

18. Scheidler J, Hricak H, Yu KK, Subak L, Segal MR. Radiological evaluation of lymph node metastases in patients with cervical cancer. A meta-analysis. JAMA. 1997;278(13):1096-1101.

19. Herrera FG, Prior JO. The role of PET/CT in cervical cancer. Front Oncol. 2013;3:34. 


\section{Publish your work in this journal}

Cancer Management and Research is an international, peer-reviewed open access journal focusing on cancer research and the optimal use of preventative and integrated treatment interventions to achieve improved outcomes, enhanced survival and quality of life for the cancer patient. The journal welcomes original research, clinical \& epidemiological

studies, reviews \& evaluations, guidelines, expert opinion \& commentary, case reports \& extended reports. The manuscript management system is completely online and includes a very quick and fair peerreview system, which is all easy to use. Visit http://www.dovepress.com/ testimonials.php to read real quotes from published authors.

Submit your manuscript here: http://www.dovepress.com/cancer-management-and-research-journal 